\title{
Ensuring a future for robotic surgery in Canada
}

\author{
Rajiv K. Singal, MD, FRCSC \\ Department of Surgery, University of Toronto, Toronto, ON, Canada
}

Cite as: Can Urol Assoc J 2019;13(6):190-1. htrp://dx.doi.org/10.5489/cuai.6046

See related article on page 184

$\mathrm{T}$ here has been a remarkable evolution in the technology that we use in the surgical treatment of our patients over the last 25 years. This followed a much longer period of incremental change over most of the previous century, rooted in the principles of Halstead. At the start of my residency in the early 1990s, virtually everything we did was through a conventional incision, occasionally with consideration to organ or functional preservation, as well as cosmesis. I was fortunate to train just as the minimally invasive era began. Over the last quarter century, the evolution from laparoscopy to robotics and other novel endoscopic techniques has been remarkable. Along the way, the cost of performing surgery has continued to grow, along with healthcare costs in general, presumably to the benefit of our patients.

Using a robotics platform poses unique challenges for Canadian surgery. The use of this technology, primarily on Intuitive Surgical's DaVinci system, has become widely adopted for a variety of both oncological and non-oncological procedures throughout the developed world. Urology, and specifically our performance of robotic radical prostatectomy, has been most responsible for the growing dissemination of robotic surgery worldwide. Do the real and perceived benefits of using this technology justify the added cost? In Canada, we have had conflicting answers to this question. Health Quality Ontario (HQO), motivated to look for ways to control a significant fiscal deficit, conducted an extensive review and concluded that robot technology should not be funded publicly. ${ }^{1}$ A similar exercise in Alberta led to an opposite conclusion. The Alberta experience more heavily took into account the costs of complications and a delayed hospital course. ${ }^{2}$

In Canada, our patients have come to rightfully expect that any proposed surgical therapeutic plan will be informed by best evidence and performed in a modern operating room with any necessary equipment made available. The surgical management of stone disease has reflected this concept well. With the development of expensive digital and fiberoptic equipment, as well as intracorporeal and extracorporeal lithotripters over the last 30 years, stone disease is managed more efficiently than an open ureterolithotomy but with greater cost. Clearly, common sense overrides any impulse to go backwards. In a healthcare system that is comprehensive but with finite resources, our mandate is both to our patients and budget realities.

How then can we best adopt robotic technology and especially robotic radical prostatectomy to urological practice? In an era of active surveillance, while there has been some reduction in the number of cases performed, radical prostatectomy still remains very a commonly performed procedure. About $40 \%$ of radical prostatectomies currently are being done robotically and that percentage is growing. The Ottawa group describes 840 cases over five years performed by 12 surgeons. Given the volume currently performed across the country, any debate around whether this technology can or should be used is moot. There are simply too many programs now with active robotics programs. Our challenge then is to find a way to responsibly deploy this technology in a manner that is safe, cost-effective, and in the best interest of our patients. Sadly, to date, much of the funding around robotic surgery across Canada has been driven through philanthropy. While it is not unreasonable for capital cost for surgical equipment to be shared in this manner, supporting ongoing operational costs, especially for cancer surgery, should remain the responsibility of our provincial funding bodies

As the authors from Ottawa point out in their experience, there are potential advantages to doing a prostatectomy robotically. The HQO recommendation heavily weighted its recommendations based on one Australian study with two experienced surgeons in each arm. The downside (and cost) of a blood transfusion and length of stay were valued less in the HQO analysis. A previous HQO advisory committee had shown, in fact, some significant clinical differences in 2014. ${ }^{3}$ The Ottawa experience reasonably reflects what we might expect to see with outcomes in a real-world setting with a number of surgeons of varying experience perform- 
ing radical prostatectomy. It could likely be extrapolated to what we may expect across Ontario and Canada. Given current volumes, the surgical treatment of prostate cancer cannot be limited to a few sites. The HQO assumption that a prostatectomy can be done with equal efficiency by all surgeons is likely also flawed as one looks to the future. Patients will increasingly find their way to robotic centres and, with fewer cases done in the wider community, this will likely concentrate volume in high-volume settings. Our well-informed patients will drive this. The case-volume of the open prostatectomy surgeon will likely diminish further in this environment. The differences already noted in a realworld setting in the Ottawa experience between robotic and open prostatectomy will then continue to grow.

Furthermore, any residency program that includes a robotic platform is currently offering trainees less opportunity to gain confidence in the standard open approach as those volumes diminish. Faced with smaller volumes in the communities they choose to work in, some may then opt to refer their patients to robotic sites. As the Ottawa group suggests, even with smaller volume surgeons, robotic prostatectomy can be done with improved outcomes. A significant goal then is to ensure a critical volume of cases at a program level to defray costs such as depreciation over a larger number of cases. If one separates initial capital cost, as was shown by the Ottawa group, the cost differential is not as great as one might expect. Our own experience in partnership with Sunnybrook has taken us to almost 600 cases performed primarily by six surgeons, also with wide variations in surgeon volume. ${ }^{4}$ Allowing perhaps three or four institutions to pool the capital cost of one robot that could then be shared to scale volume may be one solution to making this technology available to the wider community. With the advent of newer, competing robotic platforms and the expiration of patents, one would anticipate that costs will further decrease.

Using a robotic surgical platform is a reality in 2019. The HQO conclusion naively assumes that robotic prostatectomy will cease to be performed as strapped funding bodies are looking for any opportunity to lower costs. Relying on the generous philanthropy of private citizens to continue to fund these programs indefinitely is not sustainable and, in reality, is a roundabout way of contributing to a growing inequity in our public healthcare systems, making patient access to care more uneven. Canadian urology can and must lead. We must continue to evaluate our own outcomes, publish Canadian data, and advocate for our patients to ensure they receive safe, comprehensive, and yes, cost-effective surgical care. A thoughtful, credible voice can responsibly ensure that robotic surgery thrives in Canada.

Competing interests: Dr. Singal reports no competing personal or financial interests related to this work.

\section{References}

1. Health Quality Ontario. Robotic surgical system for radical prostatectomy: A health technology assessment. Ont Heal Technol Assess Ser 2017;17: 1-172.

2. Health Technology and Policy Unit G of A. Robot-assisted laparoscopic prostatectomy. Alberta Heal Technol Decis Process 2017:1-488.

3. Robotic-assisted minimally invasive prostatectomy: OHTAC recommendation. January 2014;1-11.

4. Goldenberg $M$, Kerbel $B$, Singal R. Improving access to surgical innovation in the community: Implementation of shared access model in Canadian Healthcare. Can Urol Assoc J 2019 January 21; Epub ahead of print. http://dx.doi.org/10.5489/cuaj.5248

Correspondence: Dr. Rajiv K. Singal, Department of Surgery, University of Toronto, Toronto, ON, Canada; rajivsingal@me.com 